\title{
Milky Way versus Andromeda: a tale of two disks
}

\author{
J. Yin ${ }^{1,5}$, J. L. Hou ${ }^{1}$, N. Prantzos ${ }^{2}$, S. Boissier ${ }^{3}$, R. X. Chang ${ }^{1}$, S. Y. Shen ${ }^{1}$, and B. Zhang ${ }^{4}$ \\ 1 Key Laboratory for Research in Galaxies and Cosmology, Shanghai Astronomical Observatory, CAS, 80 Nandan Road, Shanghai, \\ 200030, PR China \\ e-mail: houj1@shao.ac.cn \\ 2 CNRS, UMR7095, Institut d'Astrophysique de Paris, 75014 Paris, France \\ 3 Laboratoire d'Astrophysique de Marseille, BP8, Traverse du Siphon, 13376 Marseille Cedex 12, France \\ ${ }^{4}$ Department of Physics, Hebei Normal University, 113 Yuhua Dong Road, Shijiazhuang, 050016, PR China \\ 5 Graduate School, the Chinese Academy of Sciences, Beijing, 100039, PR China
}

Received 10 April 2009 / Accepted 22 June 2009

ABSTRACT

\begin{abstract}
Aims. We study the chemical evolution of the disks of the Milky Way (MW) and of Andromeda (M31), to identify the common properties and differences between the two major galaxies of the Local Group.

Methods. We use a large set of observational data for M31, including observations of the star formation rate (SFR) and gas profiles, as well as stellar metallicity distributions along its disk. When expressed in terms of the corresponding disk scale lengths, we show that the observed radial profiles of MW and M31 exhibit interesting similarities, suggesting the possibility of a description within a common framework.

Results. We find that the profiles of stars, gas fraction, and metallicity of the two galaxies, as well as most of their global properties, are well described by our model, provided that the star formation efficiency in M 31 disk is twice as high as in the MW. We show that the star formation rate profile of M31 cannot be described by any form of the Kennicutt-Schmidt law (KS Law) for star formation. We propose that these discrepancies are caused by the fact that M 31 has an active star formation history in the recent past, consistent with the hypotheses of a "head-on" collision with the neighboring galaxy (most probably M32) about 200 Myr ago.

Conclusions. The MW has most probably experienced quiescent secular evolution, making possible a fairly successful description with a simple model. If M 31 is more typical of spiral galaxies, more complex models, involving galaxy interactions, will be required for the description of spirals.
\end{abstract}

Key words. Galaxy: disk - Galaxy: formation - galaxies: evolution - Local Group - galaxies: individual: M 31 - galaxies: abundances

\section{Introduction}

Substantial progress has been made in understanding the evolution of disk galaxies in a cosmological context, both with analytical models (Mo et al. 1998; Efstathiou 2000) and with numerical simulations, by adopting much higher resolution and including more and more physical ingredients, such as dark matter profiles and assembly, baryon accretion histories, gas cooling and supernova feedback, and multi-phase interstellar medium (e.g., Cole et al. 2000; Samland \& Gerhard 2003; Monaco 2004; Kang et al. 2005; Heller et al. 2007; Guo \& White 2008; Roškar et al. 2008). However, the complexity of the relevant baryonic physics is not yet fully understood and no satisfactory disk model at present exists, although most recent simulations of high resolution and improved treatment about stellar feedback have succeeded in producing disks resembling observed ones (Governato et al. 2007; Mayer et al. 2008).

Because of those difficulties, the simple phenomenological models developed in the eighties and nineties may still be of considerable help. In those models, the complex processes related to disk formation through gas accretion (e.g., merging histories, gas cooling, stellar feedback) are simply described by an analytical infall law. These models do not construct the galaxy "ab initio" but rely on the observed present-day features of a galaxy to infer its past history, thus they have been characterized as "backwards" models (e.g., Ferreras \& Silk 2001). They have been widely used in studies of the chemical evolution of the Milky Way (e.g., Matteucci \& Francois 1989; Prantzos \& Aubert 1995; Chiappini et al. 1997; Prantzos \& Silk 1998; Boissier \& Prantzos 1999; Chang et al. 1999, 2002; Cescutti et al. 2007 etc.) allowing important progress towards our understanding of our Galaxy's evolution. Some agreement has been reached among the various groups concerning e.g.,: i) the necessity of substantial infall on long timescales (to explain the local G-dwarf metallicity distribution and the small degree of astration of deuterium); ii) the necessity of radially varying timescales for the infall and the star formation rate (to obtain the observed profiles of metallicity, gas fraction, and colors); and iii) no need to vary IMF or strong galactic winds.

As one of the three disk galaxies in the Local Group, Andromeda (M31 or NGC224) provides a unique opportunity for testing theories of galaxy formation and evolution (Klypin et al. 2002; Widrow et al. 2003; Widrow \& Dubinski 2005; Geehan et al. 2006; Tamm et al. 2007; Tempel et al. 2007). The wealth of available data can be used to constrain models of the evolution of the disk, bulge and halo of M 31 . However, because of its size, proximity and large bulge, most work has been completed on the stellar and kinematic properties of M 31 halo and globular clusters (Beasley et al. 2004; Burstein et al. 2004; Durrell et al. 2004; Chapman et al. 2006; Font et al. 2008; Koch et al. 2008; Lee et al. 2008), outer disk (Ibata et al. 2005; 
Irwin et al. 2005; Worthey et al. 2005; Brown et al. 2006, 2007, 2008; Richardson et al. 2008) and the central bulge (Jacoby \& Ciardullo 1999; Salow \& Statler 2004; Sarajedini \& Jablonka 2005; Davidge et al. 2006; Olsen et al. 2006).

Star formation (SF) histories in various regions of the M 31 disk and halo have also been measured with the Hubble Space Telescope (HST) and ground-based large telescopes. For example, Williams (2003a, b) has measured the star formation history in several regions of the M 31 disk from the KPNO/CTIO Local Group survey and found that the total mean star formation rate for the disk is about $1 M_{\odot} \mathrm{yr}^{-1}$. With deep HST photometry, Bellazzini et al. (2003) and Worthey et al. (2005) studied the stellar abundance distributions and star formation history in many locations of the disk (see also Ferguson \& Johnson 2001; Sarajedini \& van Duyne 2001; Williams 2002; Brown et al. 2006; Olsen et al. 2006). Those observations inferred that the M 31 disk has a mean disk age of around 6-8 Gyr and mean metallicity of $[\mathrm{Fe} / \mathrm{H}] \sim-0.2$, albeit with substantial spread in both cases.

Compared with the Milky Way, M 31 appears to have been more active in the past, although its current star formation rate is lower than that of our Galaxy. Based on a survey of spiral properties, Hammer et al. (2007) suggested that the Milky Way is a rather quiescent galaxy, atypical of its class, while the M 31 may be closer to a typical spiral. Using detailed two components (disk+halo) chemical evolution models, Renda et al. (2005) compared some chemical properties of the M31 and MW disk/halo, and concluded that M 31 must have a higher star formation efficiency and/or shorter infall timescale. Deep photometry of the M 31 halo shows that it hosts populations of old and metal-poor stars, along with younger ones and of higher metallicity, pointing to a prolonged period of active merging (Brown 2009 and references therein). The two ring-like structures observed in M 31 (Block et al. 2006) are interpreted as the result of a recent $(<200 \mathrm{Myr}$ ago) collision with a companion galaxy (Block et al. 2006) and infer that M31 has experienced recent merging.

In this paper, we attempt a comparative study of the chemical evolution of MW and M31, by constraining our model with a more extended data set than in any previous work. Our data include global properties and radial profiles of gas, stars, gas fraction, star formation rate, and oxygen abundances, as well as stellar metallicity distributions along the disk of M31 (Sect. 2). We find that, when the radial profiles are expressed in terms of the corresponding scalelengths of the stellar disks, the MW and M 31 present some interesting similarities (Sect. 2.4) encouraging us to adopt a single phenomenological model for the description of both galaxies (Sect. 3). The model describes fairly well all of the key properties of MW and most of M 31 (Sect. 4), provided the star formation efficiency is twice as large in the latter case. We discuss the successes and failures of the model, and we compare to previous work in Sect. 5. Section 6 summarizes our results.

\section{Observational properties}

\subsection{Stellar disks: scalelengths and masses}

The stellar disks of Milky Way and M31 are well described by exponential surface density profiles, given by

$\Sigma_{*}\left(r, t_{\mathrm{g}}\right)=\Sigma\left(r_{0}, t_{\mathrm{g}}\right) \mathrm{e}^{-\left(r-r_{0}\right) / r_{\mathrm{d}}}$,

where $r_{\mathrm{d}}$ is the disk scalelength and $\Sigma\left(r_{0}, t_{\mathrm{g}}\right)$ is the local surface density at some distance $r_{0}$ from the galactic center at the present time $t_{\mathrm{g}}=13.5 \mathrm{Gyr}$. In the case of the Milky Way, the reference distance is obviously the Sun's distance of $r_{0}=R_{\odot \mathrm{MW}}=$ $8 \mathrm{kpc}$, where the local stellar surface density is evaluated to be $\Sigma\left(r_{0}, t_{\mathrm{g}}\right)=37 M_{\odot} \mathrm{pc}^{-2}$ (Flynn et al. 2006). The total stellar mass of the disk is then given by

$M_{\mathrm{d}}=\int_{r_{\mathrm{b}}}^{r_{2}} 2 \pi r \Sigma_{*}\left(r, t_{\mathrm{g}}\right) \mathrm{d} r$,

where $r_{\mathrm{b}}=2.5 \mathrm{kpc}$ is the bulge radius and $r_{2}$ the outer disk radius.

Observed disk scalelengths are obtained from measurements of surface brightness profiles in various wavelength bands and are wavelength dependent. A $B$ band scalelength reflects mostly the SFR profile in the past $\sim 1$ Gyr, while $K$ or $R$ scalelengths reflect the total stellar population, cumulated over the age of the disk.

For the Milky Way disk, we adopt the mean value of $r_{\mathrm{d}}=$ $2.3 \pm 0.6 \mathrm{kpc}$ (from measurements in the $R$ or $I$ bands), derived from the compilation of Hammer et al. (2007). The total mass up to $15 \mathrm{kpc}$ is then $\sim 3 \times 10^{10} M_{\odot}$.

By adding $\sim 0.7 \times 10^{10} M_{\odot}$ for the gaseous disk mass (as estimated in the next section) one derives a total baryonic disk mass of $\sim 3.7 \times 10^{10} M_{\odot}$, in good agreement with mass models of the Milky Way (e.g., Dehnen \& Binney 1998; Naab \& Ostriker 2006).

Based on the observed disk surface brightness of M31, Walterbos \& Kennicutt $(1987,1988)$ measured the disk scalelength at different wavelengths. They obtained $r_{\mathrm{d}}=6.8,5.8,5.3$, and $5.2 \mathrm{kpc}$ in the $U, B, V$, and $R$ bands, respectively. Worthey et al. (2005) obtained $5.6 \mathrm{kpc}$ in the $I$ band, while for $K$ band Hiromoto et al. (1983) found $r_{\mathrm{d}}=4.2 \mathrm{kpc}$. With IRAC onboard the SPITZER space telescope, Barmby et al. (2006) measured, for the first time, the mid-infrared surface brightness profile of M 31 and found a scalelength of $6.08 \mathrm{kpc}$ in the $L$ band. We note that different authors adopt different distance scales for M 31. In Table 1, we list all the available observed disk scalelengths and scale them to the same distance of $785 \mathrm{kpc}$ (McConnachie et al. 2005). Overall, the values are consistent for different bands, except at shorter wavelengths that are likely to be affected by dust extinction. In this paper, we adopt an averaged value from four observed values from three bands $(R, I, K)$, which is $r_{\mathrm{d}}=$ $5.5 \mathrm{kpc}$. This value is within the range of $r_{\mathrm{d}}=5.8 \pm 0.4 \mathrm{kpc}$ found in Hammer et al. (2007).

The total mass of M 31 disk is obtained from observational data and mass models. In their disk-bulge-halo model, Widrow et al. (2003) found that their best-fit model requires the M31 disk mass (stars + gas) to be about $7 \times 10^{10} M_{\odot}$. The mass model of Geehan et al. (2006) also infers a similar disk mass of $\sim 7.2 \times$ $10^{10} M_{\odot}$, by adopting a disk mass-to-light ratio of 3.3. In this paper, we adopt the M 31 total disk mass of $M_{\text {tot }}=7 \times 10^{10} M_{\odot}$. By subtracting a gas mass of $\sim 6 \times 10^{9} M_{\odot}$ (see next section), we obtain a total disk stellar mass of $5.9 \times 10^{10} M_{\odot}$ for Andromeda.

In summary, the M31 disk is about 2 times as massive and 2.4 times as large as the Milky Way disk.

\subsection{Gas and SFR profiles}

The present-day profiles of gas and star formation provide strong constraints on models of the chemical evolution of a galactic disk. In the case of the Milky Way, relevant observational data were collected in Boissier \& Prantzos (1999), and we adopt those data in this work (Fig. 1, left panels). The gaseous profile is characterized by a broad peak at a galactocentric distance 
Table 1. Observation of Milky Way and M 31 (re-scaled to $785 \mathrm{kpc}$ ).

\begin{tabular}{|c|c|c|c|c|}
\hline Observable & Milky Way & Reference & M31 & Reference \\
\hline \multicolumn{5}{|l|}{ Global properties } \\
\hline Type & SbcI-II & 1 & SbI-II & 1 \\
\hline$K$-band & $M_{K}=-24.02$ & 2 & $M_{K}=-24.70$ & 3 \\
\hline \multicolumn{5}{|l|}{ Total luminosity } \\
\hline$L_{B}\left(10^{10} L_{B \odot}\right)$ & 1.8 & 4 & 3.3 & 5 \\
\hline$L_{V}\left(10^{10} L_{V \odot}\right)$ & 2.1 & 6 & $2.6 \sim 2.7$ & 1 \\
\hline$L_{K}\left(10^{10} L_{K \odot}\right)$ & 5.5 & 7 & $6 \sim 12^{a}$ & 8 \\
\hline Total colour $(B-V)$ & 0.84 & 4 & 0.81 & 9 \\
\hline \multicolumn{5}{|l|}{ Mass } \\
\hline Total $\left(10^{10} M_{\odot}\right)$ & $40-55$ & 10,11 & $107-140$ & 12 \\
\hline Visible $\left(10^{10} M_{\odot}\right)$ & 5.0 & 3 & $5.9-8.7$ & 5 \\
\hline \multicolumn{5}{|l|}{ Rotational curve $\left(\mathrm{km} \mathrm{s}^{-1}\right)$} \\
\hline Flat velocity & 220 & 1 & 226 & 13 \\
\hline \multicolumn{5}{|l|}{ Bulge } \\
\hline Stellar mass $\left(10^{10} M_{\odot}\right)$ & $1-2$ & 14 & 3.2 & 15 \\
\hline Effective radius (kpc) & 2.5 & 1 & 2.6 & 1 \\
\hline \multicolumn{5}{|l|}{ 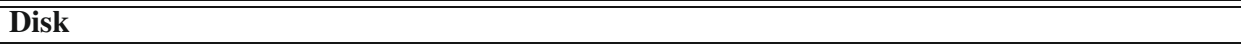 } \\
\hline \multicolumn{5}{|l|}{ Scalelength (kpc) } \\
\hline$U$ & & & 7.7 & 9 \\
\hline$B$ & $4.0 \sim 5.0$ & 7 & 6.6 & 9 \\
\hline$V$ & $2.5 \sim 3.5$ & 6,16 & 6.0 & 9 \\
\hline$R$ & 2.3 & 3 & 5.9 & 9 \\
\hline I & & & 5.7 & 17 \\
\hline$K$ & $2.3 \sim 2.8$ & 18 & 4.8 & 19 \\
\hline$L$ & & & 6.08 & 8 \\
\hline Total SFR $\left(M_{\odot} \mathrm{yr}^{-1}\right)$ & $\sim 1-5$ & 20,21 & $0.35-1.0$ & $1,8,21,22$ \\
\hline Infall rate $\left(M_{\odot} \mathrm{yr}^{-1}\right)$ & $0.5 \sim 5$ & 21,23 & & \\
\hline \multicolumn{5}{|l|}{ Total mass of } \\
\hline $\operatorname{disk}\left(10^{10} M_{\odot}\right)$ & $3.5^{b}$ & 24 & $\sim 7$ & $15,25,26$ \\
\hline $\operatorname{star}\left(10^{10} M_{\odot}\right)$ & $3.0^{c}$ & 16 & $\sim 6$ & 5 \\
\hline $\operatorname{gas}\left(10^{10} M_{\odot}\right)$ & $\sim 0.7$ & 27,28 & $\sim 0.6$ & 29 \\
\hline $\mathrm{HI}\left(10^{10} M_{\odot}\right)$ & 0.4 & 1 & $\sim 0.5$ & $1,29,30$ \\
\hline $\mathrm{H}_{2}\left(10^{10} M_{\odot}\right)$ & 0.11 & 31 & $\sim 0.02-0.04$ & $29,30,31$ \\
\hline Gas fraction & $\sim 0.15-0.2$ & 20, This paper & $\sim 0.09$ & This paper \\
\hline \multicolumn{5}{|l|}{ Abundance gradient } \\
\hline \multicolumn{5}{|l|}{ Colour gradient } \\
\hline$B-V\left(\mathrm{mag} \mathrm{kpc}^{-1}\right)$ & & & 0.016 & 9 \\
\hline
\end{tabular}

Note: ${ }^{a}$ : Assuming $M / L_{K}=1.15\left(M / L_{K}\right)_{\odot}$ and M 31 mass is taken to be $(7-14) \times 10^{10} M_{\odot} ;{ }^{b}$ : derived based on the disk scalelength $r_{\mathrm{d}}=2.3 \mathrm{kpc}$ and total disk surface density at the solar neighborhood $\Sigma_{\text {tot }}=50 M_{\odot} \mathrm{pc}^{-2} ;{ }^{c}$ : derived based on the stellar disk scalelength $r_{\mathrm{d}}=2.3 \mathrm{kpc}$ and stellar surface density at the solar neighborhood $\Sigma_{*}=37 M_{\odot} \mathrm{pc}^{-2}$.

Reference: (1) van den Bergh (1999); (2) Drimmel \& Spergel (2001); (3) Hammer et al. (2007); (4) van der Kruit (1986); (5) Tamm et al. (2007); (6) Sackett (1997); (7) Kent et al. (1991); (8) Barmby et al. (2006); (9) Walterbos \& Kennicutt (1988); (10) Xue et al. (2008); (11) Sakamoto et al. (2003); (12) Tempel et al. (2007); (13) Carignan et al. (2006); (14) Dehnen \& Binney (1998); (15) Geehan et al. (2006); (16) Zheng et al. (2001); (17) Worthey et al. (2005); (18) Freudenreich (1998); (19) Hiromoto et al. (1983); (20) Boissier \& Prantzos (1999); (21) Fraternali (2009); (22) Williams (2003)a; (23) Blitz et al. (1999); (24) Holmberg \& Flynn (2004); (25) Klypin et al. (2002); (26) Widrow et al. (2003); (27) Dame (1993); (28) Kulkarni \& Heiles (1987); (29) Nieten et al. (2006); (30) Dame et al. (1993); (31) Koper et al. (1991); (32) Deharveng et al. (2000); (33) Daflon \& Cunha (2004); (34) Rudolph et al. (2006); (35) Smartt et al. (2001); (36) Trundle et al. (2002).

4-5 kpc (caused by the "molecular ring" present at this distance) and the SFR profile is also concentrated towards the inner disk. The total gas mass is estimated to be $\sim 7 \times 10^{9} M_{\odot}$ and the total star formation rate is SFR $\sim 1-3 M_{\odot} \mathrm{yr}^{-1}$ (e.g., Boissier \& Prantzos 1999, and references therein).

For M 31, the observed radial profiles for $\mathrm{HI}$ and $\mathrm{H}_{2}$ gas surface densities (Berkhuijsen 1977; Walterbos 1986; Koper et al. 1991; Loinard et al. 1999) allow us to establish the radial gas profile displayed in Fig. 1 (right top). It is also characterized by a broad peak, located at a galactocentric distance twice as large as in the case of the MW. The HI profile from these studies is also consistent with the one measured by Chemin et al. (2009).

The star formation rate in several regions of M31 disk has been carefully measured by both ground-based photometry and
Hubble Space Telescope (Bellazzini et al. 2003; Williams 2002, 2003a,b; Brown et al. 2006; Olsen et al. 2006). The current total SFR of the M 31 disk is estimated to be $0.4 \sim 1 M_{\odot} \mathrm{yr}^{-1}$ (Williams 2003a,b; Barmby et al. 2006), i.e., less than half of the value in the Milky Way disk; this shows that M31 is currently a rather quiescent galaxy.

By analyzing data from the GALEX UV satellite, it is now possible to obtain SFR radial profiles for a number of local galaxies derived not from $\mathrm{H} \alpha$ data, but from the UV continuum (Boissier et al. 2007). In Fig. 1, we show the adopted gas (upper panels) and SFR (lower panels) profiles of the Milky Way and M 31 disks. From this figure, we see that the two spirals have quite different properties in their gas and SFR profiles in the inner part of the disk (between 3 and $7 \mathrm{kpc}$ ). The Milky Way has 


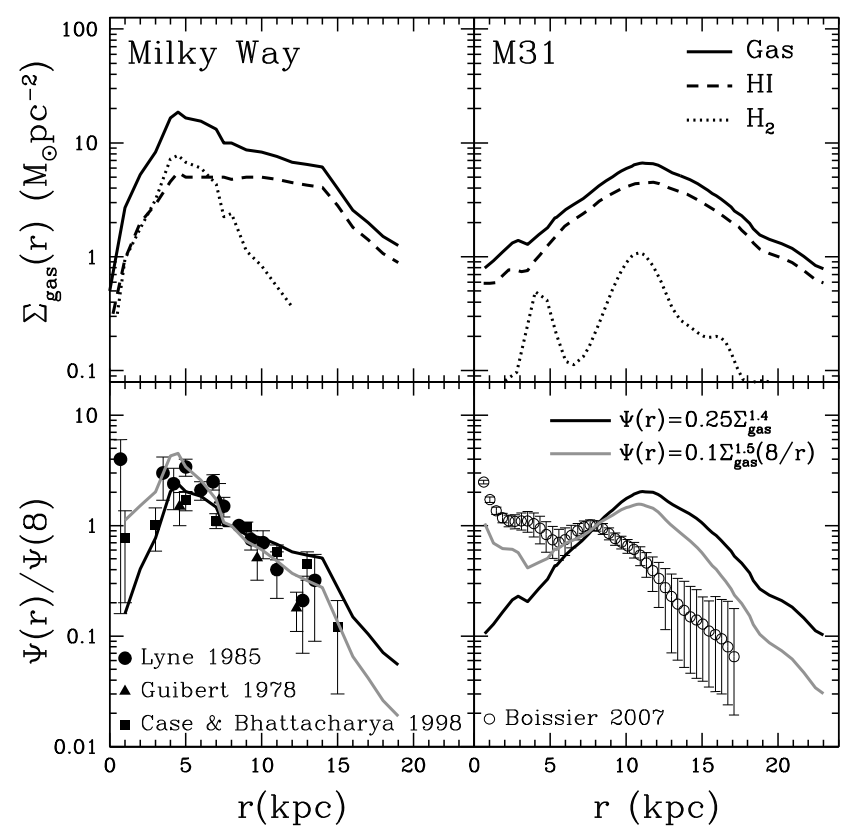

Fig. 1. Observed profiles of the gas and star formation rate for the Milky Way and M31 disks. Upper panel: current surface density profiles of HI, molecular gas, and total gas in the Milky Way and M31. Lower panel: observed estimations of SFR of the Milky Way (filled symbols, from Boissier \& Prantzos 1999) and M 31 disks (open symbols from Boissier et al. 2007). The lines in the lower panels are the results calculated according to the star formation law given in the right panel.

more gas in this region, while M31 has most of its gas outside that region.

The total gas mass of the disks is obtained by integrating the gas profiles from Fig. 1 for both galaxies, starting from the inner disk boundary (assumed to be at the bulge radius $r_{\mathrm{b}} \sim 2.5 \mathrm{kpc}$ for the MW and $r_{\mathrm{b}} \sim 5 \mathrm{kpc}$ for M31) outwards. We find $M_{\text {gas,Mw }} \sim 7 \times 10^{9} M_{\odot}$ and $M_{\text {gas,M } 31} \sim 6 \times 10^{9} M_{\odot}$ (average value, taking uncertainties into account). Since the M31 disk is twice as massive as the Milky Way disk, its global gas fraction is about $1 / 2$ that of the Milky Way disk ( 0.09 versus 0.19 , respectively). This implies that the disk of M31 had an overall higher star formation efficiency than the Milky Way (assuming that they have similar ages).

UV studies of star formation with GALEX have shown that, in general, the correlation between SFR and gas surface density is compatible with empirical Kennicutt (1998a,b) SFR laws, with some scatter at low surface densities. However, as Boissier et al. (2007) show, this correlation fails for some individual galaxies, and especially for M 31 (see their Fig. 6). In the lower two panels of Fig. 1, we also show the expected behaviour of two different SFR laws. The first depends only on gas surface density, according to

$\Psi(r)=0.25 \Sigma_{\text {gas }}^{1.4}(r)$,

on the basis of the observational data of Kennicutt (1998b, hereafter KS Law). The second depends on both gas surface density and radius and is motivated by the idea that star formation is induced by spiral waves moving around a rotating disk (e.g., Wyse \& Silk 1989, see also Sect. 3.2):

$\Psi(r) \propto \frac{\sum_{\text {gas }}^{n}(r)}{r}$

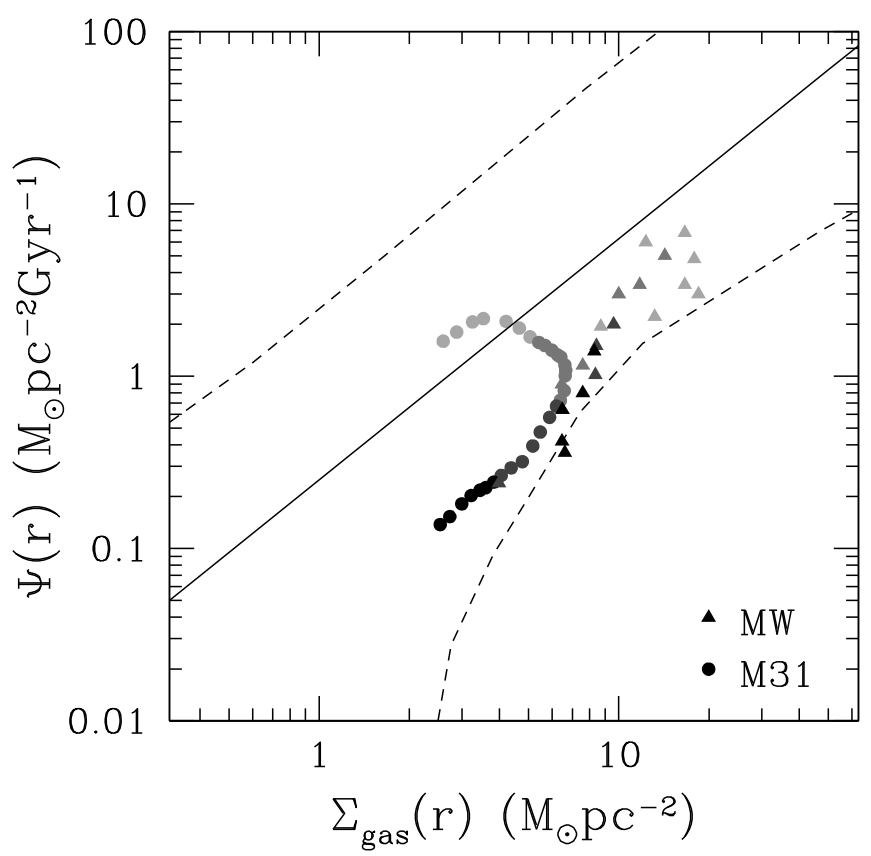

Fig. 2. Relationship between observed gas surface density and star formation rate surface density for M 31 (filled circles) and Milky Way disks (filled triangles). Full line is the classical Kennicutt law (Eq. (3)). Regions between two dashed lines indicate the results for a number of local galaxies observed by GALEX (from Boissier et al. 2007). The M 31 shows an untypical path in its inner region, while the Milky Way shows a rather normal behaviour.

For the MW disk, the observed gas and SFR profiles are found to be reproduced well by both SFR laws; as a result, the total SFR is also readily reproduced. However, for M 31 , none of the SFR laws brings agreement between observed gas and SFR profiles: there is a great difference between theoretical expectations and observations in the inner part of the disk (with little gas but, curiously, high observed SFR). As a result, any attempt to fit the SFR of the inner disk with one of the aforementioned SFR laws will lead to an overestimate of the SFR in the outer disk.

To demonstrate further the different behaviour of local star formation rate in M 31 and Milky Way disks, we plot the relationship between the observed gas surface density and star formation rate surface density in Fig. 2. We also show the general trend of SFR with gas surface density (within dashed curves) for a number of nearby galaxies (from Boissier et al. 2007). It is clear that M 31 has a peculiar behaviour compared to both the Milky Way and other local galaxies, especially in its inner region, where high star formation rate corresponds to low amount of gas. In the range $7-11 \mathrm{kpc}$, the SFR decreases as the gas surface density increases, in contrast to the classical SFR law. Beyond $11 \mathrm{kpc}$, both SFR and amount of gas decrease with radius, roughly in agreement with the Kennicutt law.

If observations of SFR in M 31 are not heavily distorted by incorrect extinction corrections, then one may conclude that the current SFR in that galaxy does not obey one of the classical star formation laws (Schmidt, Kennicutt, or some modified form of them). Star formation in M31 is maybe(or has been) perturbed by some external event, e.g., a major recent encounter with a galaxy of the Local Group. Observations of a two-ringlike structures by Block et al. (2006) are interpreted as evidence that a nearly central head-on encounter with a companion galaxy (probably M 32) occurred about 200 Myr ago. If this is indeed 
the case, then the present day SFR profile of M 31 cannot be used as a constraint on the chemical evolution model, since the perturbation induced in the gaseous disk by the collision most probably affected, for (at least) one orbital time, the SFR in M31. Timeintegrated observable, such as e.g., the total stellar profile or the abundance profile (and, to a lesser extent, the gaseous profile) certainly remain valid constraints.

\subsection{Disk abundance gradients}

Abundance gradients are an essential ingredient in an accurate picture of galaxy formation and evolution (Boissier \& Prantzos 1999; Hou et al. 2000; Chiappini et al. 2001; Hou et al. 2002; Cescutti et al. 2007; Magrini et al. 2009; Fu et al. 2009). The existence of abundance gradients along the MW disk has been established using different tracers (Hou \& Chang 2001). However, the size of this gradient is still debated. Thus, oxygen or/and iron abundance gradients of between about -0.06 and $\sim-0.07$ dex $\mathrm{kpc}^{-1}$ are obtained by using tracers as HII regions and B stars (Rudolph et al. 2006 and references therein), planetary nebulae (Maciel et al. 2006; Maciel \& Costa 2008), and open clusters (Chen et al. 2003, 2008). However, values about $40 \%$ smaller are obtained by using those same tracers, e.g., Deharveng et al. (2000, with HII regions), Daflon \& Cunha (2004, using several tracers), and Andrievsky et al. (2004, with Cepheids).

The situation for the abundance gradient in the disk of M 31 is also far from clear. Early observations (Dennefeld \& Kunth 1981; Blair et al. 1982) used HII regions and supernova remnants. A value of $\operatorname{dlog}(\mathrm{O} / \mathrm{H}) / \mathrm{d} r=-0.06 \pm 0.034 \mathrm{dex} \mathrm{kpc}^{-1}$ was derived using nebular emission line ratios by Galarza et al. (1999). The main uncertainty originates in the empirical calibrations used to derive the electronic temperatures in the nebular phase. By reanalysing earlier data from various authors, Trundle et al. (2002) found smaller values for the oxygen abundance gradient, ranging from $-0.027 \mathrm{dex} \mathrm{kpc}^{-1}$ down to $-0.013 \mathrm{dex} \mathrm{kpc}^{-1}$. Furthermore, their analysis of five B-type supergiants covering the galactocentric distances of 5-12 kpc inferred to a negligible oxygen abundance gradient of $-0.006 \pm$ $0.02 \mathrm{dex} \mathrm{kpc}^{-1}$; in contrast, they found a slightly more significant gradient for $\mathrm{Mg}$, of $-0.023 \pm 0.02 \mathrm{dex} \mathrm{kpc}^{-1}$.

In summary, the abundance gradient in M31 is very poorly known at present. We adopt here a value of $-0.017 \mathrm{dex} \mathrm{kpc}^{-1}$, i.e., the mean between the most extreme values of $-0.027 \mathrm{dex} \mathrm{kpc}^{-1}$, and $-0.006 \mathrm{dex} \mathrm{kpc}^{-1}$ found in the various analysis of Trundle et al. (2002). We note that this value is substantially smaller (factor 3-4) than that of the Milky Way disk (-0.07 dex kpc $\left.\mathrm{kp}^{-1}\right)$, but only by a factor of 2 if the value of $-0.04 \mathrm{dex} \mathrm{kpc}^{-1}$ is adopted for our Galaxy. Finally, if we express the abundance gradients in terms of corresponding scalelengths ( $\mathrm{dex} / r_{\mathrm{d}}$ ), then the scaled gradient of M 31 disk is found to be two times smaller (equal) than that of the MW disk for the cases of -0.07 dex kpc ${ }^{-1}\left(-0.04 \mathrm{dex} \mathrm{kpc}^{-1}\right)$.

Figure 3 displays the observed oxygen abundance profiles in the Milky Way and M31 disks. In the case of MW, two values of the gradient are shown, corresponding to -0.07 dex $\mathrm{kpc}^{-1}$ (circles) and $-0.04 \mathrm{dex} \mathrm{kpc}^{-1}$ (solid line), respectively.

\subsection{A unified description of the Milky Way and M31}

Table 1 summarizes the main observational features of the MW and M 31 disks. The different sizes of the two major galaxies of the Local Group ensure that a direct comparison between their

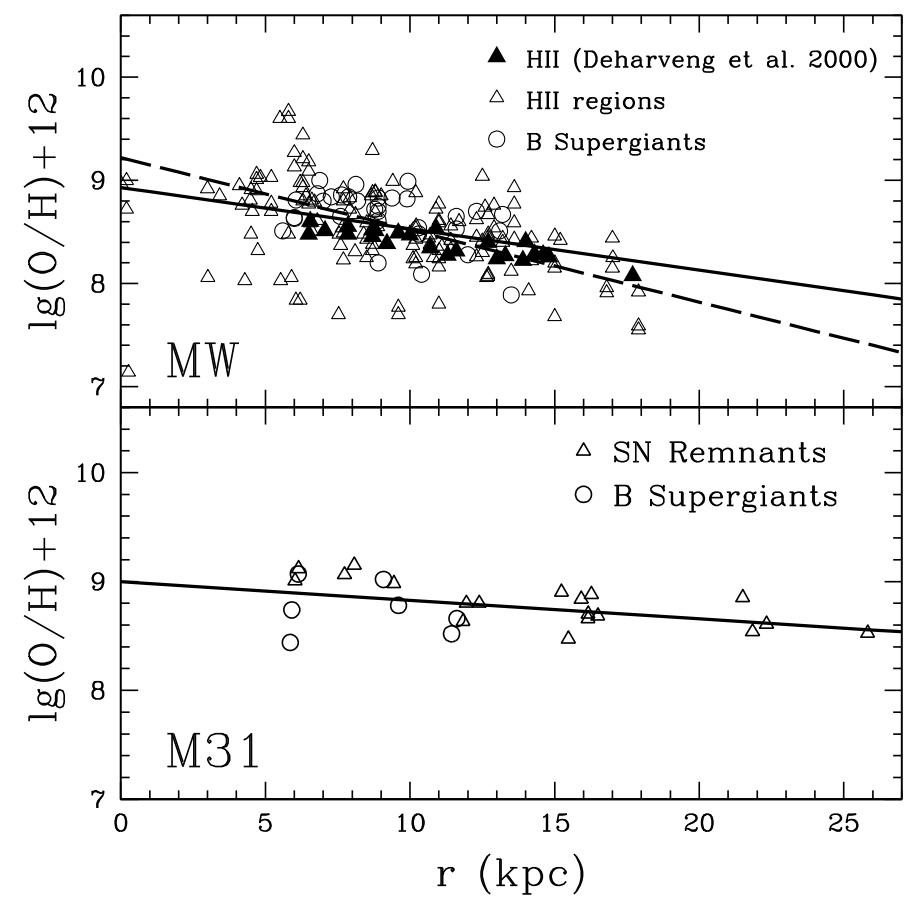

Fig. 3. Observed oxygen abundance gradient in the Milky Way (top, data from Rudolph et al. 2006; Deharveng et al. 2000) and M31 (bottom, data from Dennefeld \& Kunth 1981; Blair 1982; Trundle et al. 2002). In the top panel, the two commonly referred values of $-0.07 \mathrm{dex} \mathrm{kpc}-1$ and $-0.04 \mathrm{dex} \mathrm{kpc}^{-1}$ appear as dashed and solid line respectively.

radial profiles is difficult, thus giving no information about the physical ingredients required to describe successfully both disks. To obtain a coherent picture, we attempt here a more physical description, by expressing all distances in terms of the corresponding disk scalelengths.

In Fig. 4, we plot the radial profiles of gas, star, SFR, gas fraction, and the oxygen abundance gradients for the two disks, using their scalelengths as distance units. It can be seen that:

(1) the gaseous profiles (top left) are rather similar, in the sense that they both display a broad peak at $\sim 2$ scalelengths from their centers. The MW has a more extended gaseous profile (in terms of scalelength);

(2) the Milky Way disk is more compact than M 31, since it has a higher stellar surface density at a given $r_{\mathrm{d}}$ value (middle left).

(3) The profiles of scaled gas fractions (middle right) of the two galaxies are quite similar in the inner disks. However, the overall gas fractions of the two disks are quite different, the MW having a gas fraction that is twice as high as that of M31;

(4) the scaled abundance gradients between the two disks are similar if we adopt the smaller reported value for the MW disk (bottom left).

Thus, when the observed profiles are expressed in terms of scalelengths, the two disks exhibit some similarities in their properties. One may then hope to be able to describe both disks with a single chemical evolution model, by varying as few as possible of the relevant parameters. We describe such a model in the next section. 

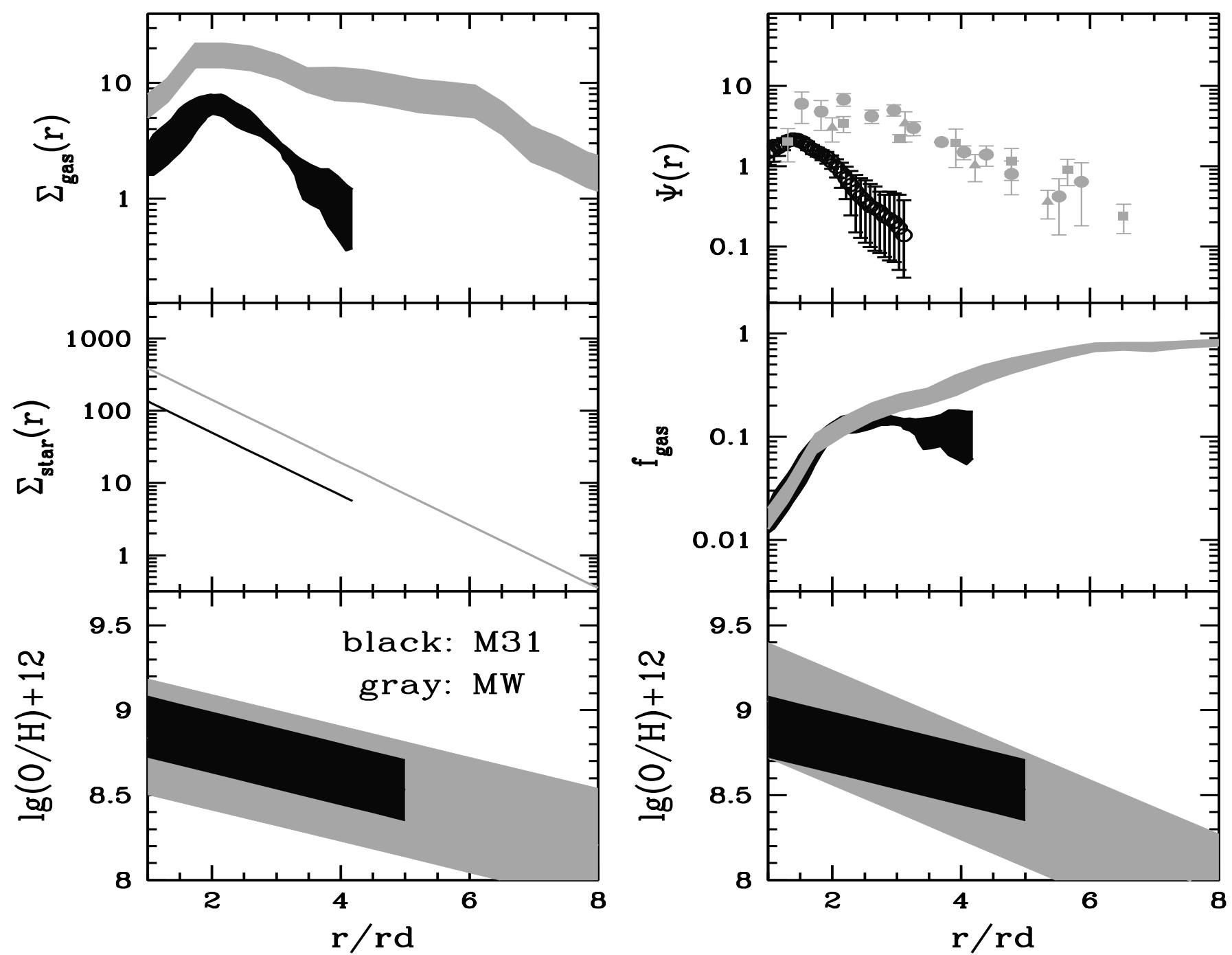

Fig. 4. Observed profiles for Milky Way and M 31 disks, with radius expressed in units of the corresponding scalelengths $r_{\mathrm{d}}$. For the abundance gradient of the Milky Way disk, two sets of values are plotted, one is -0.07 dex kpc ${ }^{-1}$ (bottom right panel), the other is $-0.04 \mathrm{dex} \mathrm{kpc}^{-1}($ bottom left panel). In the latter case, MW and M 31 have similar abundance gradients, when expressed in dex $/ r_{\mathrm{d}}$. Shaded areas are the typical observed scatter.

\section{The model}

In the case of the Milky Way, models with radially dependent infall and star formation laws, forming the disk inside-out, are generically used (Prantzos \& Aubert 1995; Boissier \& Prantzos 1999; Hou et al. 2000; Chiappini et al. 2001; Magrini et al. 2009). These models reproduce several of the salient observational features of the MW disk (including the abundance gradients), albeit with different levels of success.

In this section, we present briefly our chemical evolution model that is similar to the one adopted successfully in the past for the Milky Way disk (see details in Boissier \& Prantzos 1999, 2000; Hou et al. 2000).

\subsection{IMF and stellar yields}

The initial mass function (IMF) $\Phi(m)$ describes the mass distribution of newborn stars and can be inferred from the observed luminosity function on the basis of the mass-to-light ratio for stars. Similar to our previous works, we adopt the IMF from the work of Kroupa et al. (1993, KTG93), where some complex factors (such as stellar binarity, ages, and metallicities, as well as mass-luminosity and colour-magnitude relationships) are explicitly taken into account (Boissier \& Prantzos 1999, 2000; Hou et al. 2000).

Stellar yields are taken from Woosley \& Weaver (1995, WW95) for massive stars, and from van den Hoek \& Groenewegen (1997, vdHG97) for low and intermediate mass stars (mass from 0.8 to $8 M_{\odot}$ ). They are all metallicity dependent.

To account for the additional source of Fe-peak elements, required to explain the observed decline in the $\mathrm{O} / \mathrm{Fe}$ abundance ratio (with increasing metallicity) in the Milky Way disk (Goswami \& Prantzos 2000), we utilize the yields of SNIa from the exploding Chandrashekhar-mass $\mathrm{CO}$ white dwarf models W7 and W70 of Iwamoto et al. (1999). These are updated versions of the original W7 model of Thielemann et al. (1986), calculated for metallicities $Z=Z_{\odot}(\mathrm{W} 7)$ and $Z=0$ (W70), respectively.

\subsection{Infall rate and timescale}

We assume that the MW and M31 disks are progressively built up by the infall of cooling primordial gas from their dark haloes. 
The form of the time dependence of the infall rate is unknown at present. In Prantzos \& Silk (1998), an asymmetric Gaussian infall rate was assumed, on the basis of dynamical arguments. However, simpler parametrizations usually are adopted, i.e., the infall rate decreases exponentially with time

$f(t, r)=A(r) \mathrm{e}^{-t / \tau(r)}$,

where $A(r)$ is a normalizing function and can be obtained by

$\int_{0}^{t_{\mathrm{g}}} A(r) \cdot \mathrm{e}^{-t / \tau(r)} \mathrm{d} t=\Sigma_{\mathrm{tot}}\left(r, t_{\mathrm{g}}\right)$,

where $\Sigma_{\text {tot }}(r, t g)$ is the current total mass profile and $\tau(r)$ is the infall timescale which is radially dependent. In the Milky Way disk, the characteristic infall timescale in the solar neighborhood $\left(R_{\odot \mathrm{MW}}=8 \mathrm{kpc}\right)$ is assumed to be $\sim 7 \mathrm{Gyr}$ (Chiappini et al. 1997; Boissier \& Prantzos 1999; Chang et al. 1999, 2002), to reproduce the local G-dwarf metallicity distribution.

The radial dependence of the infall timescale for the MW disk is given by $\tau_{\mathrm{MW}}(r)=b r / r_{\mathrm{d}}$, where $r_{\mathrm{d}}$ is the scalelength and $b$ is a free parameter. Positive values of $b$ imply an inside-out formation of the disk and we adopt here $b=2.5$, which infers formation timescales of $\sim 2 \mathrm{Gyr}$ for the inner disk and $\sim 10 \mathrm{Gyr}$ for the outer disk.

In the case of M31, we adopt the prescription used in Boissier \& Prantzos (2000), according to which the infall timescale is assumed to be correlated with both surface density and galaxy mass

$\tau^{-1}(r)=\tau_{\mathrm{MW}}^{-1}(r)+0.4\left(1.0-\frac{V_{C}}{220}\right)$,

where $V_{C}$ (in $\mathrm{km} \mathrm{s}^{-1}$ ) is the flat rotational velocity for the galaxy disk and $\tau_{\mathrm{MW}}(r)$ (in Gyr) is the infall timescale for the Milky Way disk. According to Hammer et al. (2007), $V_{C}$ for M 31 is about $226 \mathrm{~km} \mathrm{~s}^{-1}$, i.e., the same as that of Milky Way disk. Therefore, our adopted prescription leads to similar infall timescale laws for both disks.

\subsection{Star formation}

The star formation rate remains the major unknown in chemical evolution studies. Kennicutt (1998a,b) found that the global SFR of disks and circumnuclear starburst galaxies is correlated with the local gas density over a wide range of surface density and SFR per unit area, spanning 5 orders in magnitude. Over that range, the empirical SFR versus gas surface density relation can be fitted by a simple power law with index $n \sim 1.4$. Kennicutt (1998a,b) also found that the data can be reproduced equally well as a function of the local dynamical timescale $\tau_{\text {dyn }}$ by $\Psi \propto \frac{\Sigma_{\text {gas }}}{\tau_{\text {dyn }}} \propto \Sigma_{\text {gas }} \Omega$, where $\Omega$ is the rotation speed of the gas. Since $\Omega \sim V(r) / r$, the SFR could be expressed as

$\Psi(r) \propto \Sigma_{\text {gas }} \frac{V(r)}{r}$,

where $V(r)$ is the circular velocity at radius $r$. Since spiral galaxies follow the relation $V(r) \sim$ constant, one derives a modified Kennicutt-Schmidt law (hereafter M-KS law), as suggested on theoretical grounds by Wyse \& Silk (1989, see also Prantzos \& Aubert 1995).

Boissier \& Prantzos (1999) adopted the index $n$ of the MKS SFR law to be $n=1.5$ on an empirical basis, to derive the present day profiles of the MW SFR (Fig. 1, bottom left). They also adopted this M-KS law in subsequent models for external
Table 2. Model parameters.

\begin{tabular}{|c|c|c|}
\hline General & $\begin{array}{l}\text { Prescription } \\
\end{array}$ & Parameter \\
\hline IMF & KTG1993 & \\
\hline Mass limits & $(0.1-100) M_{\odot}$ & \\
\hline SFR & $\alpha \Sigma_{\text {gas }}^{1.5}\left(r_{\text {eq } \odot} / r\right)$ & $\alpha$ \\
\hline Stellar yields & vdHG97,WW95 & \\
\hline Metallicity of infall gas & $Z_{f}=0$ & \\
\hline Infall timescale & $\tau(r)=b\left(r / r_{\mathrm{d}}\right)$ & $b$ \\
\hline Age of disk (Gyr) & 13.5 & \\
\hline Individual & Milky Way & M31 \\
\hline Scalelength $r_{\mathrm{d}}(\mathrm{kpc})$ & 2.3 & 5.5 \\
\hline Equivalent $r_{\mathrm{eq} \odot}(\mathrm{kpc})$ & 8.0 & 19.0 \\
\hline Total disk mass $\left(10^{10} M_{\odot}\right)$ & 5.0 & 7.0 \\
\hline$V_{\text {rot }}\left(\mathrm{km} \mathrm{s}^{-1}\right)$ & 220 & 226 \\
\hline
\end{tabular}

spirals, which can successfully reproduce most of the chemical and photometric properties of disk galaxies (Boissier \& Prantzos 2000; Boissier et al. 2001) and in particular the observed abundance gradients (Prantzos \& Boissier 2000). Fu et al. (2009) used both the KS law and M-KS law to predict the time evolution of the Galactic disk abundance gradient. By comparing the model predictions with the observed results from open clusters and planetary nebulae with different ages, they concluded that by adopting the M-KS law, model results are more consistent with the observed evolution of abundance gradient. Therefore, we adopt this M-KS law for Milky Way and M 31 disks:

$\Psi(r)=\alpha \Sigma_{\text {gas }}^{1.5}\left(\frac{r_{\text {eq } \odot}}{r}\right)$.

The coefficient $\alpha$ is related to the star formation efficiency. All other things being equal, it appears that the star formation efficiency in M31 must be at least twice as high as in the MW, since its observed gas fraction is twice as small (Table 1 and discussion in Sect. 2.2). We shall see in the next section that this larger $\alpha$ is required to fit the $\mathrm{M} 31$ data.

\section{Model results and comparison with both the Milky Way and M 31 disks}

We run our simulations with the parameters of Table 2 for the MW and M31 disks. We note that we adopt the same model parameters for both galaxies, hence attempt to describe them in a unified framework, except for: (i) the small difference in their infall rate from Eq. (7), which infers that M 31 is slightly older than the MW; and (ii) the star formation efficiency parameter $\alpha$, assumed here to be twice as large for M31 as for the MW, i.e., $\alpha_{\mathrm{MW}}=0.1$ and $\alpha_{\mathrm{M} 31}=0.2$.

\subsection{Radial profiles}

In Fig. 5, we show the model predictions of the radial profiles for gas, stars, SFR, gas fraction, and oxygen at time $t=13.5 \mathrm{Gyr}$, and we compare them with observational data. The first two columns display results for MW and M 31, respectively, as a function of radius $r$ expressed in $\mathrm{kpc}$. The third column presents the same results on a common scale of normalized radius $r / r_{\mathrm{d}}$ for both galaxies; this allows us to visualize the similarities and differences between the two disks more clearly.

The main results of the comparison with observations can be summarized as follows:

1) In both cases, exponential disk profiles are naturally obtained, since most of the infalling gas (the radial profile of 


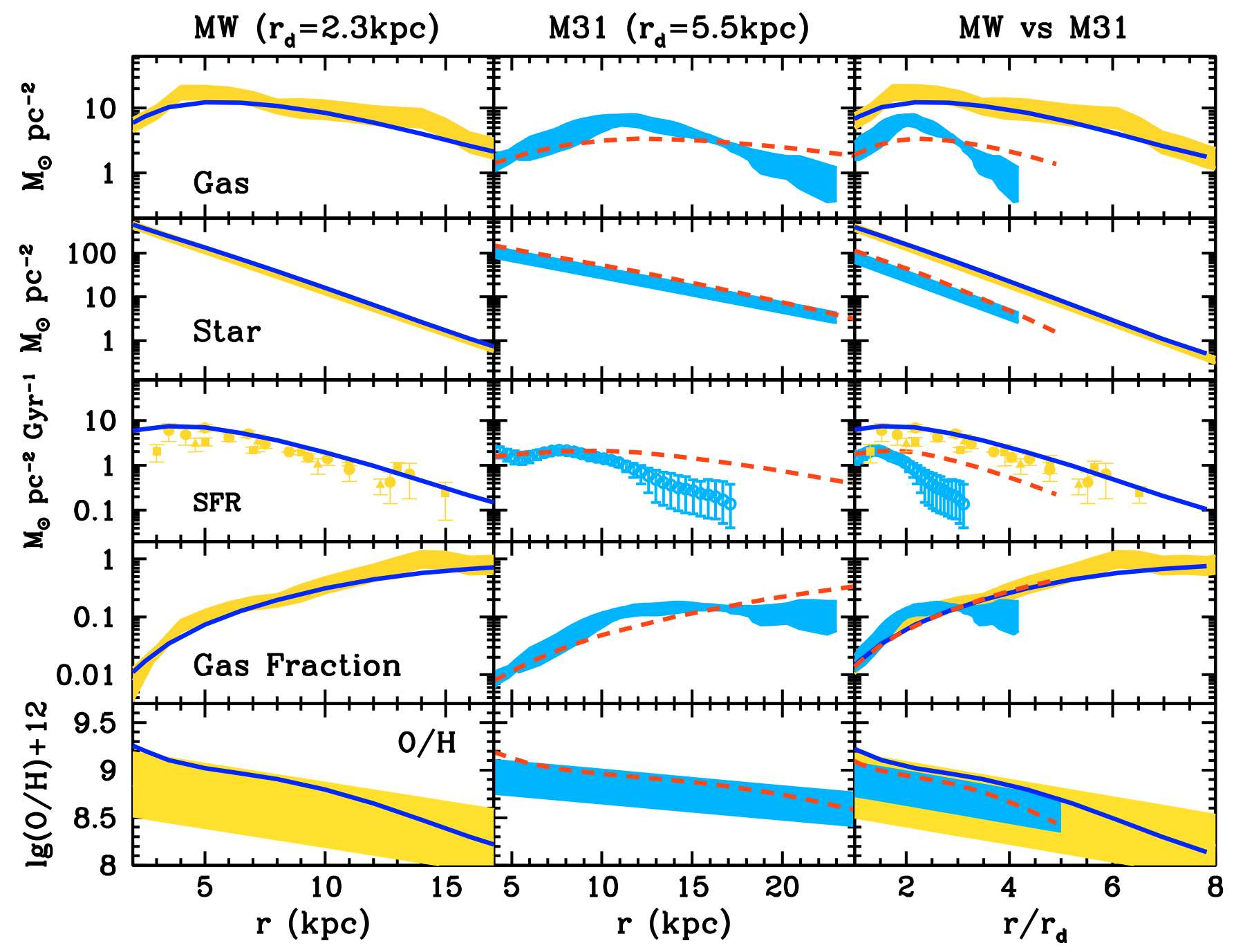

Fig. 5. Current profiles of gas, stars, SFR, gas fraction, and oxygen abundance (from top to bottom) for MW and M 31 . In the left and middle panels, profiles for MW and M31, respectively, are expressed in terms of physical radius $r$ (in $\mathrm{kpc}$ ); in the right panels, profiles for both disks are expressed in terms of normalised radius $r / r_{\mathrm{d}}$. Observations are presented as yellow shaded (for MW with $-0.04 \mathrm{dex} / \mathrm{kpc}$ ) or blue shaded (for M 31 ) areas and model results by solid (for the MW) and dashed (for M 31) curves, respectively.

which is normalised by means of Eq. (6)) is converted into stars.

2) The model gaseous profiles reach a broad maximum, attained at the observed position, approximately at two scalelengths from the galactic centers. This maximum is obtained in the models by means of the radial dependence of the SF efficiency (being greater in the inner disk, it produces a gas fraction profile $f_{\text {gas }}(r)$ that increases with radius, see next paragraph) and the total surface density profile $\Sigma_{\text {tot }}(r)$, which is constrained to decrease with radius. The gaseous profile is the product of the two, i.e., $\Sigma_{\text {gas }}(r)=f_{\text {gas }}(r) \Sigma_{\text {tot }}(r)$, so the resulting curve goes through a maximum, and within our unified scheme this happens at $\sim 2 r_{\mathrm{d}}$.

3) The gas fraction profile decreases monotonically inwards, in perfect agreement with observations for the MW and in fair agreement for the inner disk of M31. The model predicts slightly higher than observed gas fractions only for the outer disk of M31. We note that, in terms of normalized radius $r / r_{\mathrm{d}}$, the gas fraction profiles of the two disks are very similar, which explains their successful description by our unified model. We also note that, in terms of physical radius, M 31 has a smaller gas fraction than the MW at a given $r$, which explains the need for a higher SF efficiency in that case. The situation is less satisfactory in the outer disk of M 31, where the gas fraction is overestimated by our model.

4) Our model predicts correctly the present day SFR profile of MW, but fails completely in the case of M31, and in particular in the outer disk of M 31. As already noticed (Sect. 2.2) the observed SFR versus gas relationship in M 31 cannot be reproduced by any form of the KS laws. Our result reflects just this impossibility. As already argued (last paragraph of Sect. 2.2), we believe that the observed SFR is affected by recent perturbations of the gaseous disk of M31, e.g., the collision with a nearby galaxy suggested by Block et al. (2006).

5) The resulting abundance gradients are compatible with observations for both MW and M31. The predicted abundance profile of MW is somewhat steeper than in the case of M 31, which is not a surprise because the two disks do not have the same scale. At the same distance from the galactic center, they have different amounts of gas and SFRs. As a result of its larger scalelength, the gas is presently more wide spread in M31 than in the Milky Way, its a resulting gas fraction rising less steeply in Andromeda, producing correspondingly a flatter abundance gradient. When we express 


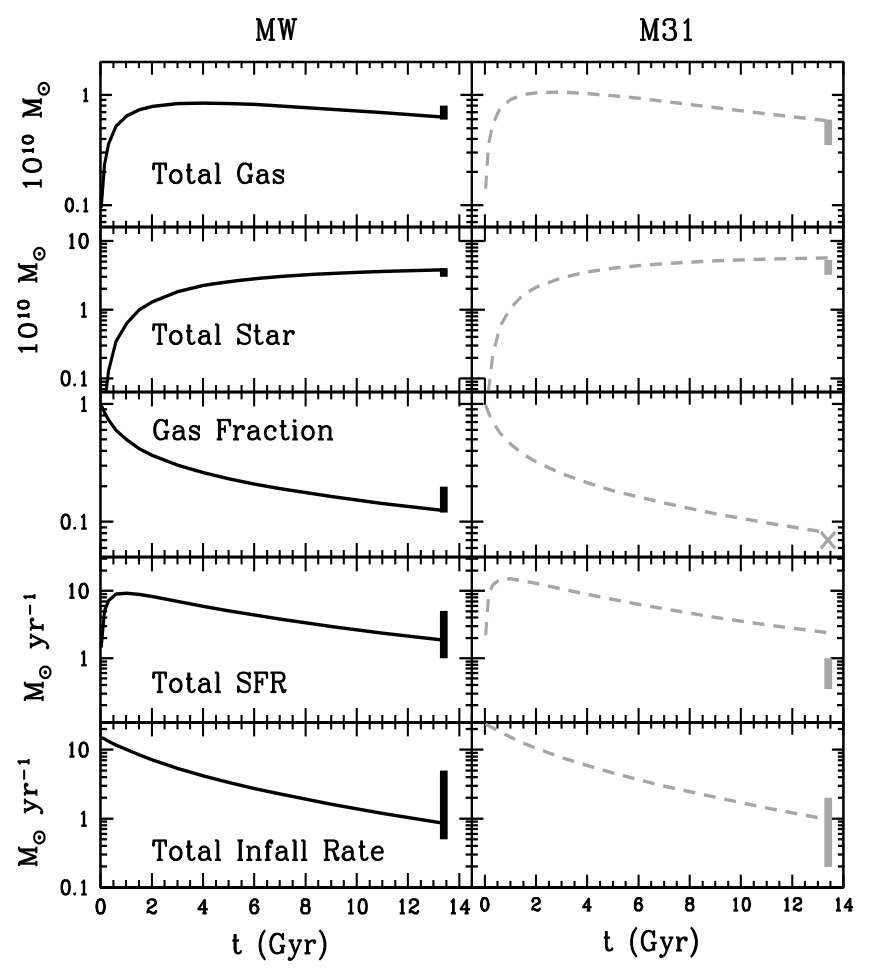

Fig. 6. Time evolution of global gas, star, gas fraction, infall rate, and SFR in M31 and Milky Way disks. The disk parameters are given in Table 2, and the coefficients $\alpha$ of SFR in Table 2 for the Milky Way and M 31 disks are $\alpha_{\mathrm{MW}}=0.1, \alpha_{\mathrm{M} 31}=0.2$, respectively. Infall timescale is $\tau(r)=2.5 r / r_{\mathrm{d}}$. Bar in the right of each plot gives the observed estimations. It can be seen that the model predicts too high a present value of SFR for M 31 disk.

the model abundance gradient in terms of their scalelengths, we obtain similar value for the two disks. In any case, taking into account all the uncertainties mentioned in Sect. 2.3, we consider the overall agreement as satisfactory. Further observations will hopefully establish the true abundance profiles of MW and M31 with greater accuracy, and perhaps allow us to develop different prescriptions for our unified model.

\subsection{Infall and star formation history}

In Fig. 6, we show the evolution of the total amount of gas, stars, SFR, and infall rate, and of the gas fraction for the disks of MW and M 31. Reasonable agreement with observations is obtained for all those quantities in the case of MW disk; this agreement results from the adopted normalization of the total disk mass and the adopted star formation efficiency. In the case of M31, the model predicts a current global SFR 2.0 $M_{\odot}$ $\mathrm{yr}^{-1}$, which is substantially higher than observational estimates (Williams 2003a,b; Barmby et al. 2006). In view of the discussion in Sect. 2.2, we do not consider this discrepancy as being significant: star formation in M 31 may have been perturbed considerably by external effects (i.e., collision with another galaxy), which are unaccounted for in our model. We also note that the current infall rate is poorly constrained for MW and virtually unconstrained for M31. In the case of M31, Thilker et al. (2004) found that an extensive population of HI clouds exists in the outskirts of the galaxy. The values displayed in Fig. 6 are in the range of $0.2-2 M_{\odot} \mathrm{yr}^{-1}$, the former being the typical value inferred from observations of accreting cold gas in

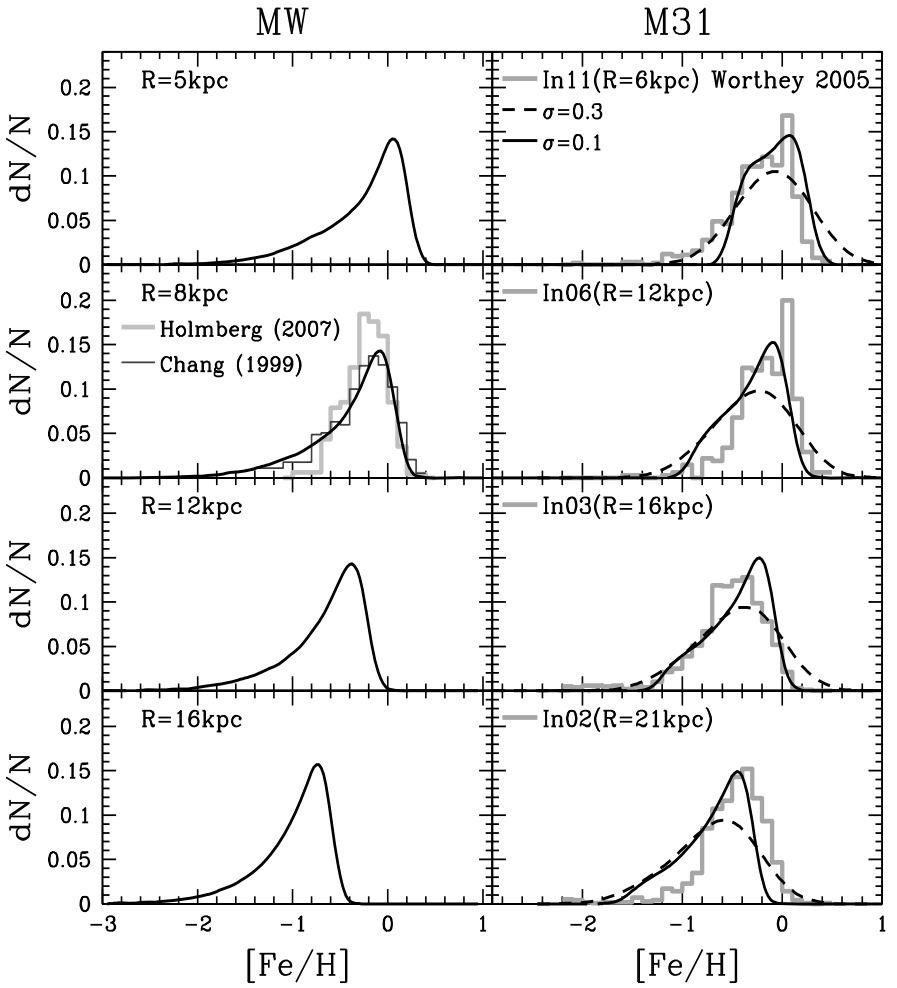

Fig. 7. The metallicity distribution functions of various regions in the Milky Way and M31 disks. The observed data in the solar neighborhood of the Milky Way disk come from Holmberg et al. (2007) and Chang et al. (1999), and the data of M 31 come from Worthey et al. (2005). The model predictions are plotted as smooth curves, after convolution with a Gaussian error function with $\sigma=0.1$ dex (full lines) and 0.3 dex (dashed lines). For M 31, we construct metallicity distributions for the age-range of RGB stars observed by Worthey et al. (2005) (stellar ages between 6 and $12 \mathrm{Gyr}$ ).

disks (Sancisi et al. 2008) and the latter from a simple theoretical argument, namely that these values are required to maintain a quasi-constant SFR over disk history for MW-size disks.

\subsection{Metallicity distributions}

We now compare the model metallicity distribution functions (MDFs) with available observations for various regions of the Milky Way and M31 disks. The model results correspond to main sequence stars with lifetimes $\tau>10 \mathrm{Gyr}$, and they have been convolved with Gaussian error functions with $\sigma=0.1 \mathrm{dex}$ (Fig. 7).

For the Milky Way disk, the observed data in the solar neighborhood are from the GK survey (Holmberg et al. 2007, who revised estimates in Nordström et al. 2004), which includes ages, metallicity, and kinematic properties for about $14000 \mathrm{~F}$ and $\mathrm{G}$ dwarfs. MDFs in other regions are unavailable at present, but future surveys, e.g., SDSS/SEGUE (Ivezić et al. 2008) and China's LAMOST project (Zhao et al. 2006), are also expected to provide information on those regions. As expected (from the adopted infall rate) our model fits rather well, albeit not perfectly, the local MDF. We note that we compare with the data of Holmberg et al. (2007) corrected for the scale height of stellar populations (dashed curve in their Fig. 22, right panel): indeed, our results concern the full extent of the so-called "solar cylinder" at $8 \mathrm{kpc}$ from the Galactic center, while local surveys are complete only within a limited volume centered on the Sun. 
However, the corrections in Holmberg et al. (2007) are made after some assumptions about the star formation history of the local disk, which is not necessarily the same as the SF history in our model. Thus, it should not be surprising that the fit is not perfect.

In the case of M31, data are available for the MDF in various places along its disk (Bellazzini et al. 2003; Worthey et al. 2005; Chapman et al. 2006). Right panel histograms are results from Worthey et al. (2005), who observed 11 regions from the inner regions to the outer disk along the major axis of M31. The median abundances in each observed field increase steadily from the inner to the outer disk. The mean stellar metallicity is $\sim 0.2$ dex lower than the gas-phase abundance (Fig. 3) in the same location.

In the right panels of Fig. 7, we also present the model predictions for the MDFs in the same radial positions as the data available for M 31. Our results are in broad agreement with observations and they reproduce the decrease of mean stellar metallicity with radius, as a consequence of the star formation and infall schemes adopted for the disk. We conclude then that, to a first approximation, M31 evolved inside-out, as expected for a normal spiral. Despite this, rather satisfactory agreement, between the data and our model, we would like to emphasize the need for more data on the MDFs of M 31 as a function of radial position in order to further constrain the evolution of its disk.

We note that when plotting the model-predicted MDFs for M 31 disk, we have assumed an error about of 0.1 dex based on Worthey et al. (2005). This assumption is self-consistent with observations since we used the data from Worthey et al. (2005). However, this adopted error in the photometric metallicity may be too small because we know that even for the situation of the halo, where the age spread should be smaller than in the disk (and associated uncertainties lower) a comparison of spectroscopic and photometric metallicities for RGB stars in M 31 should scatter about \pm 0.3 dex (see Kalirai et al. 2008). Therefore, we also indicate the model MDFs for the M 31 disk with photometric errors of 0.3 dex by dashed lines in Fig. 7. As expected, the model-predicted MDFs are wider than the observed distributions in this case, but the peak position is roughly the same.

We emphasize that while calculating the models, the results are for disk evolution with full star formation history, that is, includes all stars in the disk with all ages. Worthey et al. (2005) did not discuss the age spread in detail, but they claimed that the age spread for their RGB stars is about 6-12 Gyr (Sect. 2, last paragraph in Worthey et al.). Therefore, we construct the metallicity distributions for M 31 disk with the age range of RGB stars observed by Worthey et al. (stellar ages between 6 and 12 Gyr) and compare them to their observations in Fig. 7.

On the other hand, Koch et al. (2005) found that for the Carina dSph, there is a larger age spread (from 2 Gyr to more than 11 Gyr), while its colour-magnitude diagram shows a narrow RGB distribution. The large age spread implies that Carina $\mathrm{dSph}$ must have undergone various episodes of star formation process. This is different from the smaller age spread reported by Worthey et al. (2005). We understand that this uncertainty calls for a more work on the observational side about the metallicity distribution of M 31 disk.

\section{Discussion}

Early works on the parallel modeling of MW and M 31 (Diaz \& Tosi 1984) found some similarities between the evolutionary properties of the two disks, but they were performed at an epoch when limited observational data provided little constraint on the models (for instance, Diaz \& Tosi 1984 compared their model to M31 data available only in the 5-11 kpc region). A more detailed comparison to observations was completed by Molla et al. (1996), who used a multi-parameter model and reproduced several features of the M 31 disk successfully.

Renda et al. (2005) focuses on MW and M31, benefitting from a larger data set and found some similarities to our work. The disks are constructed inside-out by slow infall and the adopted SFR is $\Psi=\alpha \Sigma_{\text {Gas }}^{2} / r$, i.e., with an exponent $n=2$ instead of 1.5 in our case. By adopting exactly the same SFR law for MW and M31, Renda et al. (2005) found that the gaseous profile is over predicted in M 31 (their model M31a), hence the need to increase their SF efficiency $\alpha$ by a factor of 2 to improve the fit to the data (their model M31b). If they had noticed the lower gas fraction in M31, they would have anticipated the problem (see our discussion in Sect. 3.3). Our conclusion based on our models agree both in terms of a higher SF efficiency in M31 compared to the MW as well as the resulting abundance gradients (smaller in the case of M31), when radius in all radially dependent terms is expressed in e.g., kpc. For some unclear reason, our model reproduces the gaseous profile of M 31 more successfully, perhaps because of our smaller exponent $n=1.5$ in the adopted SFR. Finally, both our model and theirs fail to reproduce observational data for the outer disk of M 31. It is difficult to continue the comparison further, because Renda et al. (2005) do not provide SFR and stellar or gas fraction profiles. The latter are mandatory in any work on chemical evolution, since they constrain, more than anything else the combined history of star formation and infall.

Despite their simplicity (independently evolving rings, no cosmological framework), models such as that presented here can provide some interesting physical insights into the evolution of MW and M31, based both on their successes and failures. The success in reproducing simultaneously the profiles of gas, SFR, and metallicity in the MW, as well as its global properties (gas fraction, total SFR, and colours, the latter being discussed in Boissier \& Prantzos 1999) implies that the overall history of the Milky Way cannot have been very different from the one found here, i.e., a slow, inside-out disk formation. However, similar solutions may, perhaps, be obtained by some other combinations of SFR and infall rate, i.e., the problem may well be degenerate, thus no firm conclusions can be drawn on each one of those two key ingredients.

In the case of M31, it is clear that a higher star formation efficiency is required, as inferred from its gas fraction, lower by a factor of $\sim 2$ than in the MW. This was already found by Renda et al. (2005), while Hammer et al. (2007) went one step further, to suggest that the MW is a particularly "quiescent" disk galaxy (for its mass) and M 31 may be closer to an average large spiral. This "quiescence" of the MW may be due to its relative isolation, while M 31 may have undergone a larger number of (and/or more important) interactions with neighboring galaxies. This picture is in line with the finding of Block et al. (2006), namely that M 31 has undergone a major interaction about 200 Myr ago; no such interaction appears to have occurred in the case of the MW over the past few billions of years.

Our formalism allows us to describe in a unified framework the properties of both the MW and M31, by using the same expression for the radial dependence of the SFR in both cases. This description is required because of the similarity between the radial profiles of the two disks, when they are expressed in terms of their respective scalelengths (Sect. 2.4). However, it is not clear whether the higher SF efficiency of M31 is caused by an external factor (i.e., more frequent/important interactions of that 
galaxy) or to an internal one (e.g., its mass, as argued in Boissier $\&$ Prantzos 2000). Applying this formalism to other disk galaxies for which large data basis are available (work in progress) will help us to clarify the situation.

On the other hand, the failure of both this work and Renda et al. (2005) to reproduce satisfactorily the gaseous profile of M31, and because we over-predict the global SFR of M31, as well as its outer SFR profile, the properties are affected considerably by recent interactions. Thus, they cannot be predicted by such simple models (unless more parameters are introduced). If this is true, and if M 31 is really closer to a typical disk (as Hammer et al. 2007 suggest), then the cosmological framework will be mandatory for the description of galactic disks; simple models, such as this one, will be able to describe successfully only the most quiescent disks, such as the MW.

\section{Summary}

We have studied the chemical evolution of the disk of M 31, using a model that had already been applied to the study of the Milky Way (Boissier \& Prantzos 1999; Hou et al. 2000). We used an extensive data set of M31 properties, including radial profiles of gas surface density, gas fraction, star formation rate, oxygen abundances, as well as metallicity distribution functions at different regions of the disk. The star formation rate profile of M 31 was derived from GALEX UV data (Boissier et al. 2007). Our main aim was to determine whether a simple chemical evolution model can successfully describe the radial and global properties of both disks.

We first summarized and compared the observational data (Sect. 2) for the two galaxies. The disk of M 31 is about 2.4 times larger and 2 times more massive than the Milky Way disk, while its gas fraction is approximately half of the one of the MW. All other things being equal, this implies that there has been a higher average star formation efficiency in M 31 disk. We found that the SF radial profile of MW is well described by "standard" SF laws, but not that of M 31 (Sect. 2.2). We attributed the later findings to a major perturbation of M 31 by a nearby galaxy, in line with the findings of Block et al. (2006). We concluded that our model (which adopts "standard" SF laws) will fail to reproduce the observed SF profile of M 31, and perhaps also the gas profile.

We found that, when radii are expressed in terms of the corresponding scalelengths, the two disks display very interesting similarities in their radial profiles (Sect. 2.4). This concerns, in particular, the gas fraction, the profile of which is quasi-identical inside the innermost two scalelengths (Fig. 4). The scaled abundance gradients of the two disks are also quite similar if we adopt for the MW the lower range of reported values (e.g., Deharveng et al. 2000; Daflon \& Cunha 2004; Andrievsky et al. 2004; Chen et al. 2008). This similarity was also found in a sample of external spirals that cover a much wider range of galaxy properties than the two disks studied here and successfully described by the models of Boissier \& Prantzos (2001). We emphasize, however, that the status of the MW abundance gradient, especially in the outer part, is still very controversial: observations show that it may not be described by a simple exponential (see e.g., Yong et al. 2005 and Carraro et al. 2007 for open cluster abundances; and Andrievsky et al. 2004 and Lemasle et al. 2008 for Cepheids). Assuming that the scaled abundance gradients are similar in MW and M31, we tried for a description of the radial properties of the two disks within the framework of a single model, which we presented in Sect. 3.

Detailed calculations illustrated that our unified model describes well all the main properties of the MW disk and most of those of M 31, provided that the SF efficiency of M 31 is adjusted to be twice as high in M 31 disk (as implied by the lower gas fraction of M31). The radial profiles of both MW and M 31 were well described, albeit less successfully in the case of M31. In particular, the model fails to match the present SFR in M 31, producing too high values in the outer disk and globally. We attributed this failure to M 31 having been perturbed recently by a major encounter, as is implied by the fact that the observed SFR profile of M 31 did not follow any form of the Kennicutt-Schmidt star formation law. On the other hand, the stellar metallicity distributions measured along the disk of M 31 reflect the disk integrated star formation during the entire history of the and should not be affected by recent events. Our model, where the bulk of Fe originates in SNIa, reproduces those distributions well, from 6 to $21 \mathrm{kpc}$.

The unified description that we have proposed here for MW and M31, by expressing their radial profiles in terms of "natural units" (the corresponding disk scalelengths), offers valuable insights into the evolution of those two disk galaxies, and our results may also be applicable to other spirals as well (work in progress).

Acknowledgements. This work is supported by the National Science Foundation of China No. 10573028, the Key Project No. 10833005, the Group Innovation Project No. 10821302, and by 973 program No. 2007CB815402.

\section{References}

Andrievsky, S. M., Luck, R. E., Martin, P., et al. 2004, A\&A, 413, 159 Barmby, P., Ashby, M. L. N., Bianchi, L., et al. 2006, ApJ, 650, L45 Beasley, M. A., Brodie, J. P., Strader, J., et al. 2004, AJ, 128, 1623 Bellazzini, M., Cacciari, C., Federici, L., et al. 2003, A\&A, 405, 867 Berkhuijsen, E. M. 1977, A\&A, 57, 9

Blair, W. P., Kirshner, R. P., \& Chevalier, R. A. 1982, ApJ, 254, 50 Blitz, L., Spergel, D. N., Teuben, P. J., et al. 1999, ApJ, 514, 818 Block, D. L., Bournaud, F., Combes, F., et al. 2006, Nature, 443, 832 Boissier, S., \& Prantzos, N. 1999, MNRAS, 307, 857 Boissier, S., \& Prantzos, N. 2000, MNRAS, 312, 398 Boissier, S., \& Prantzos, N. 2001, MNRAS, 325, 321 Boissier, S., Boselli, A., Prantzos, N., et al. 2001, MNRAS, 321, 733 Boissier, S., Gil de Paz, A., Boselli, A., et al. 2007, ApJS,173, 524 Brown T. M. 2009 [arXiv: 0901 .2577], in proceedings of "Galaxy Evolution: Emerging Insights and Future Challenges", 11-14 November 2008, Austin, USA

Brown, T. M., Smith, E., Ferguson, H. C., et al. 2006, ApJ, 652, 323 Brown, T. M., Smith, E., Ferguson, H. C., et al. 2007, ApJ, 658, L95 Brown, T. M., Beaton, R., Chiba, M., et al. 2008, ApJ, 685, L121 Burstein, D., Li, Y., Freeman, K. C., et al. 2004, ApJ, 614, 158 Carignan, C., Chemin, L., Huchtmeier, W. K., et al. 2006, ApJ, 641, L109 Carraro, G., Geisler, D., Villanova, S., et al. 2007, A\&A, 476, 217 Case, G. L., \& Bhattacharya, D. 1998, ApJ, 504, 761

Cescutti, G., Matteucci, F., Francois, P., et al. 2007, A\&A, 462, 943 Chang, R. X., Hou, J. L., Shu, C. G., et al. 1999, A\&A, 350, 38 Chang, R. X., Shu, C. G., \& Hou, J. L. 2002, ChJA\&A, 2, 226 Chapman, S. C., Ibata, R., Lewis, G. F., et al. 2006, ApJ, 653, 255 Chemin, L., Carignan, C., \& Foster, T. 2009, ApJ, submitted [arXiv:0908.0326]

Chen, L., Hou, J. L., \& Wang, J. J. 2003, AJ, 125, 1397

Chen, L., Hou, J. L., Zhao, J. L., et al. 2008, IAUS, 248, 433

Chiappini, C., Matteucci, F., Gratton, R., et al. 1997, ApJ, 477, 765

Chiappini, C., Matteucci, F., Romano, D., et al. 2001, ApJ, 554, 1044 Cole, S., Lacey, C. G., Baugh, C. M., et al. 2000, MNRAS, 319, 168 Daflon, S., \& Cunha, K. 2004, ApJ, 617, 1115

Dame, T. M. 1993, AIP Conf., 278, 267

Dame, T. M., Koper, E., Israel, F. P., et al. 1993, ApJ, 418, 730

Davidge, T. J., Jensen, J. B., \& Olsen, K. A. G. 2006, AJ, 132, 521

Deharveng, L., Peña, M., Caplan, J., et al. 2000, MNRAS, 311, 329

Dehnen, W., \& Binney, J. 1998, MNRAS, 294, 429

Dennefeld, M., \& Kunth, D. 1981, AJ, 86, 989

Diaz, A., \& Tosi, M. 1984, MNRAS, 208, 365

Drimmel, R., \& Spergel, D. N. 2001, ApJ, 556, 181

Durrell, P. R., Harris, W. E., \& Pritchet, C. J. 2004, AJ, 128, 260 
Efstathiou, G. 2000, MNRAS, 317, 697

Ferguson, A. M. N., \& Johnson, R. A. 2001, ApJ, 559, 13

Ferreras, I., \& Silk, J. 2001, ApJ, 557, 165

Flynn, C., Holmberg, J., Portinari, L., et al. 2006, MNRAS, 372, 1149

Font, A. S., Johnston, K. V., Ferguson, A. M. N., et al. 2008, ApJ, 673, 215

Fraternali, F., \& Binney, J. J. 2008, MNRAS, 386, 935

Freudenreich, H. T. 1998, ApJ, 492, 495

Fu, J., Hou, J. L., Yin, J., et al. 2009, ApJ, 696, 668

Geehan, J. J., Fardal, M. A., Babul, A., et al. 2006, MNRAS, 366, 996

Galarza, V. C., Walterbos, R. A. M., \& Braun, R. 1999, AJ, 118, 2775

Goswami, A., \& Prantzos, N. 2000, A\&A, 359, 191

Governato, F., Willman, B., Mayer, L., et al. 2007, MNRAS, 374, 1479

Guibert, J., Lequeux, J., \& Viallefond, F. 1978, A\&A, 68, 1

Guo, Q., \& White, S. D. M. 2008, MNRAS, 384, 2

Hammer, F., Puech, M., Chemin, L., et al. 2007, ApJ, 662, 322

Heller, C. H., Shlosman, I., \& Athanassoula, E. 2007, ApJ, 671, 226

Hiromoto, N., Maihara, T., Oda, N., et al. 1983, PASJ, 35, 413

Holmberg, J., \& Flynn, C. 2004, MNRAS, 352, 440

Holmberg, J., Nordström, B., \& Andersen, J. 2007, A\&A, 475, 519

Hou, J. L., \& Chang, R. X. 2001, Progr. Astron., 19(1), 68

Hou, J. L., Prantzos, N., \& Boissier, S. 2000, A\&A, 362, 921

Hou, J. L., Chang, R. X., \& Chen, L. 2002, ChJAA, 2, 17

Ibata, R., Chapman, S., Ferguson, A. M. N., et al. 2005, ApJ, 634, 287

Irwin, M. J., Ferguson, A. M. N., Ibata, R. A., et al. 2005, ApJ, 628, 105

Ivezić, Z̈., Sesar, B., Jurić, M., et al. 2008, ApJ, 684, 287

Iwamoto, K., Brachwitz, F., Nomoto, K., et al. 1999, ApJS, 125, 439

Jacoby, G. H., \& Ciardullo, R. 1999, ApJ, 515, 169

Kalirai, J. S., Gilbert, K. M., Guhathakurta, P., et al. 2008, ApJ, 648, 389

Kang, X., Jing, Y. P., Mo, H. J., et al. 2005, ApJ, 631, 21

Kennicutt, R. C. 1998a, ARA\&A, 36, 189

Kennicutt, R. C. 1998b, ApJ, 498, 541

Kent, S. M., Dame, T. M., Fazio, G., et al. 1991, ApJ, 378, 131

Klypin, A., Zhao, H. S., \& Somerville, R. S. 2002, ApJ, 573, 597

Koch, A., Wilkinson, M., Grebel, E. K., et al. 2005, Proc. IAU Colloq. 198, ed.

H. Jerjen, \& B. Binggeli (Cambridge: Cambridge University Press), 134

Koch, A., Rich, R. M., Reitzel, D. B., et al. 2008, ApJ, 689, 958

Koper, E., Israel, F. P., Dame, T. M., et al. 1991, ApJ, 383, L11

Kroupa, P., Tout, C. A., \& Gilmore, G. 1993, MNRAS, 262, 545

Kulkarni, S., \& Heiles, C. 1987, In Interstellar Processes, ed. D. Hollenbach, \&

H. Thronson (Dordrecht: Kluwer), 87

Lacey, C. G., \& Fall, S. M. 1985, ApJ, 290, 154

Lee, M. G., Hwang, H. S., Kim, S. C., et al. 2008, ApJ, 674, 886

Lemasle, B., Francois, P., Piersimoni, A., et al. 2008, A\&A, 490, 613

Loinard, L., Dame, T. M., Heyer, M. H., et al. 1999, A\&A, 351, 1087

Lyne, A., Manchester, R., \& Taylor, J. 1985, MNRAS, 213, 613

Maciel, W. J., \& Costa, R. D. D. 2008 [arXiv: 0806. 3443]

Maciel, W. J., Lago, L. G., \& Costa, R. D. D. 2006, A\&A, 453, 587

Magrini, L., Sestito, P., Randich, S., et al. 2009, A\&A, 494, 95

Matteucci, F., \& Francois, P. 1989, MNRAS, 239, 885
Mayer, L., Governato, F., \& Kaufmann, T. 2008, Adv. Sci. Lett., 1, 7

McConnachie, A. W., Irwin, M. J., Ferguson, A. M. N., et al. 2005, MNRAS, 356, 979

Molla, M., Ferrini, F., \& Diaz, A. 1996, ApJ, 466, 668

Mo, H. J., Mao, S., \& White, S. D. M. 1998, MNRAS, 295, 319

Monaco, P. 2004, MNRAS, 352, 181

Naab, T., \& Ostriker, J. P. 2006, MNRAS, 366, 899

Nieten, Ch., Neininger, N., Guélin, M., et al. 2006, A\&A, 453, 459

Nordström, B., Mayor, M., Andersen, J., et al. 2004, A\&A, 418, 989

Olsen, K. A. G., Blum, R. D., Stephens, A. W., et al. 2006, AJ, 132, 271

Prantzos, N. 2008, A\&A, 489, 525

Prantzos, N., \& Aubert, O. 1995, A\&A, 302, 69

Prantzos, N., \& Boissier, S. 2000, MNRAS, 313, 338

Prantzos, N., \& Silk, J. 1998, ApJ, 507, 229

Renda, A., Kawata, D., Fenner, Y., et al. 2005, MNRAS, 356, 1071

Richardson, J. C., Ferguson, A. M. N., Johnson, R. A., et al. 2008, AJ, 135, 1998

Roškar, R., Debattista, V. P., Quinn, T. R., et al. 2008, ApJ, 684, L79

Rudolph, A. L., Fich, M., Bell, G. R., et al. 2006, ApJS, 162, 346

Sackett, P. D. 1997, ApJ, 483, 103

Salow, R. M., \& Statler, T. S. 2004, ApJ, 611, 245

Sakamoto, T., Chiba, M., \& Beers T. C. 2003, A\&A, 397, 899

Samland, M., \& Gerhard, O. E. 2003, A\&A, 399, 961

Sancisi, R., Fraternali, F., Oosterloo, T., et al. 2008, A\&ARv, 15, 189

Sarajedini, A., \& Jablonka, P. 2005, AJ, 130, 1627

Sarajedini, A., \& van Duyne, J. 2001, AJ, 122, 2444

Smartt, S. J., Crowther, P. A., Dufton, P. L., et al. 2001, MNRAS, 325, 257

Tamm, A., Tempel, E., \& Tenjes, P. 2007 [arXiv:0707.4375v1]

Tempel, E., Tamm, A., \& Tenjes, P. 2007 [arXiv:0707.4374v1]

Thielemann, F. K., Nomoto, K., \& Yokoi, K. 1986, A\&A, 158, 17

Thilker, D. A., Braun, R., Walterbos, R. A. M., et al. 2004, ApJ, 601, L39

Trundle, C., Dufton, P. L., Lennon, D. J., et al. 2002, A\&A, 395, 519

van den Bergh, S. 1999, A\&ARv, 9, 273

van den Hoek, L. B., \& Groenewegen, M. A. T. 1997, A\&AS, 123, 305 (vdHG97)

van der Kruit, P. C. 1986, A\&A, 157, 230

Walterbos, R. A. M. 1986, BAAS, 18, 915

Walterbos, R. A. M., \& Kennicutt, R. C. 1987, A\&AS, 69, 311

Walterbos, R. A. M., \& Kennicutt, R. C. 1988, A\&A, 198, 61

Widrow, L. M., \& Dubinski, J. 2005, ApJ, 631, 838

Widrow, L. M., Perrett, K. M., \& Suyu, S. H. 2003, ApJ, 588, 311

Williams, B. F. 2002, MNRAS, 331, 293

Williams, B. F. 2003a, AJ, 126, 1312

Williams, B. F. 2003b, MNRAS, 340, 143

Woosley, S. E., \& Weaver, T. A. 1995, ApJS, 101, 181 (WW95)

Worthey, G., España, A., MacArthur, L. A., et al. 2005, ApJ, 631, 820

Wyse, R. F. G., \& Silk, J. 1989, ApJ, 339, 700

Xue, X. X., Rix, H. W., Zhao, G., et al. 2008, ApJ, 684, 1143

Yong, D., Carney, B. W., \& Teixera de Almeida, M. L. 2005, AJ, 130, 597

Zhao, G., Chen, Y. Q., Shi, J. R., et al. 2006, ChJA\&A, 6, 265

Zheng, Z., Flynn, C., Gould, A., et al. 2001, ApJ, 555, 393 